Management international

International Management

Gestiòn Internacional

L'innovation-valeur comme dynamique stratégique. Le cas du

Pentathlon Moderne

Innovation-Value as a Strategic Dynamic. The Case of the

Modern Pentathlon

Innovación-valor como una dinámica estratégica. El caso del

Pentatlón Moderno

Frédéric Lassalle

Volume 21, numéro 3, printemps 2017

URI : https://id.erudit.org/iderudit/1052771ar

DOI : https://doi.org/10.7202/1052771ar

Aller au sommaire du numéro

Éditeur(s)

HEC Montréal

Université Paris Dauphine

ISSN

1206-1697 (imprimé)

1918-9222 (numérique)

Découvrir la revue

Citer cet article

Lassalle, F. (2017). L'innovation-valeur comme dynamique stratégique. Le cas du Pentathlon Moderne. Management international / International Management / Gestiòn Internacional, 21(3), 145-156. https://doi.org/10.7202/1052771ar

\section{Résumé de l'article}

Cette recherche s'intéresse à l'existence d'une dynamique d'innovations stratégiques. Elle est étudiée au travers de l'innovation-valeur (Kim et Mauborgne, 2005) dans le cas de la fédération internationale de pentathlon moderne (UIPM). Cette instance doit innover pour assurer sa survie face à ses principales parties prenantes, le Comité International Olympique (CIO) et ses clients, les pratiquants. L'étude de cas permettra de tester l'existence d'une dynamique d'innovations stratégiques.
Tous droits réservés (C) Management international / International Management / Gestión Internacional, 2017
Ce document est protégé par la loi sur le droit d'auteur. L'utilisation des services d'Érudit (y compris la reproduction) est assujettie à sa politique d'utilisation que vous pouvez consulter en ligne.

https://apropos.erudit.org/fr/usagers/politique-dutilisation/ 


\title{
L'innovation-valeur comme dynamique stratégique. Le cas du Pentathlon Moderne
}

\author{
Innovation-Value as a Strategic Dynamic. \\ The Case of the Modern Pentathlon
}

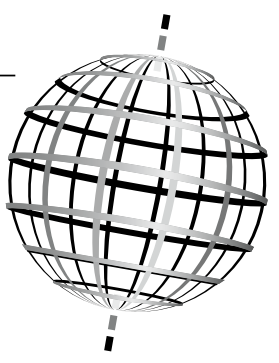

\section{Innovación-valor como una dinámica estratégica. El caso del Pentatlón Moderno}

\author{
FRÉDÉRIC LASSALLE \\ Equipe d'Accueil du CREGO EA 7317 \\ IAE Dijon Université de Bourgogne \\ Dijon, France
}

\section{RÉSUMÉ}

Cette recherche s'intéresse à l'existence d'une dynamique d'innovations stratégiques. Elle est étudiée au travers de l'innovation-valeur (Kim et Mauborgne, 2005) dans le cas de la fédération internationale de pentathlon moderne (UIPM). Cette instance doit innover pour assurer sa survie face à ses principales parties prenantes, le Comité International Olympique (CIO) et ses clients, les pratiquants. L'étude de cas permettra de tester l'existence d'une dynamique d'innovations stratégiques.

Mots clés : Innovation stratégique, Innovationvaleur, Pentathlon moderne

\section{ABSTRACT}

This research focuses on the existence of a dynamic strategic innovations. It is studied through the innovation value (Kim and Mauborgne, 2005) in the case of the International Federation of Modern Pentathlon (UIPM). This federation must innovate to survive against its major stakeholders, the International Olympic Committee (IOC) and its customers, the practitioners. The case study will test the existence of a dynamic strategic innovations.

Keywords: Strategic Innovation, Innovationvalue, Modern Pentathlon

\section{RESUMEN}

Esta investigación se centra en la existencia de una dinámica de las innovaciones estratégicas. A través de la innovaciónvalor (Kim y Mauborgne, 2005) se aborda el caso de la Federación Internacional de Pentatlón Moderno (UIPM). Esta federación debe innovar para sobrevivir frente a sus principales grupos de interés, el Comité Olímpico Internacional (COI) y sus clientes. El estudio de caso que se presenta intenta demostrar la existencia de una dinámica de las innovaciones estratégicas.

Palabras Clave: innovación estratégica, innovación-valor, Pentatlón moderno
$\mathrm{L}$ 'innovation doit être au cœur des organisations quel que soit le secteur d'activité dans lequel elles évoluent (Hamel et Prahalad, 1989). Il existe plusieurs raisons à cela : des antécédents managériaux (D’Amato et Roome, 2009; Birkinshaw, 2010; Harder, 2011), des antécédents externes (Damanpour et Aravind, 2012), des technologies (Cohen et Levinthal, 1990; Christensen, 1997; Mol et Birkinshaw, 2009; 2012), des facteurs contextuels (Nickell et al., 2001; Grant, 2008), ou des résultats (Walker et al., 2010; Gebauer, 2011). Au final, plusieurs catégories possibles d'innovations existent (Buaron, 1981; Hamel et Prahalad, 1996; Birkinshaw et al., 2008; Hamel, 2008; Roy, 2010). Dans le cas de la fédération internationale de pentathlon moderne (UIPM), l'innovation passe avant tout par un changement de procédés (prééminence opérationnelle), de produits/services (souvent en utilisant la technologie) voire de stratégie avec une innovationvaleur. L'innovation stratégique, appelée aussi rupture, évolution disruptive, innovation-valeur, stratégie océan bleu (Roy, 2010), consiste pour une organisation à changer de manière radicale les règles concurrentielles en proposant une nouvelle valeur aux clients tout en maitrisant les coûts (Lehmann-Ortega et Roy, 2009). Cette innovation stratégique va être développée par Kim et Mauborgne $(2004 ; 2005)$ et leur concept d'océan bleu.
La littérature théorique, sur l'innovation et l'innovation stratégique, est importante (Martinsons, 1993; Krinsky et Jenkins, 1997; Markides, 1997, 1998; Geroski, 1998; Schlegelmilch et al., 2003). La légitimité de cette recherche se justifie dans la mesure où la littérature théorique ne s'intéresse que peu à la notion d'innovation-valeur (Parvinen et al., 2011). Roy (2007) dans le cas des complexes cinématographiques, Čirjevskis et al. (2010) dans le cas du B to B, ou encore Burke et al. (2009) ou O'Gorman (2009) cherchent à identifier les démarches d'innovation-valeur de diverses organisations. Néanmoins, aucun d'eux ne s'intéresse à l'existence d'une dynamique stratégique lors d'une innovation-valeur. Est-il possible de réussir plusieurs innovationsvaleurs successivement au sein de la même organisation? Kim et Mauborgne (2005) ne répondent que partiellement à cette question. Pour eux, les secteurs d'activité connaissent des innovations stratégiques mais qui sont souvent le fait de différentes entreprises. Par exemple, dans le cas de l'exploitation des salles de cinéma, les évolutions (Nickelodeon, Palaces, Multiplexe, Mégaplexe) ont, à chaque fois, été proposées par une entreprise différente de l'évolution précédente (Kim et Mauborgne, 2005). La justification réside pour la littérature théorique dans la mise en place de processus et de routines (Feldman, 2003; Becker, 2004, 2005; Grodal, 2015). 
Le problème pour l'UIPM réside dans le fait que la stratégie d'innovation "océan bleu » a déjà été utilisée lors de la réorganisation des cinq disciplines de ce sport entre les JO de Pékin (2008) et ceux de Londres (2012). Cette restructuration, ainsi que quelques évolutions techniques, comme le passage du pistolet à plomb au pistolet laser, a permis à l'UIPM de proposer une innovation-valeur et ainsi de conserver sa place pour les jeux olympiques de 2016 et 2020 . Le maintien s'explique par un regroupement de sites (piscine, salle d'arme et équitation (ce dernier permet l'équitation et le combiné course-tir)). Une plus grande visibilité pour les sponsors (format plus court et plus attractif pour les médias), une plus grande lisibilité pour le spectateur et téléspectateur (le leader après les trois épreuves s'élance en premier dans le combiné et le premier à franchir la ligne est désigné vainqueur). Toutes ces évolutions tiennent compte des recommandations du CIO permettant le maintien du pentathlon moderne (PM) au sein du programme olympique. Cette innovation-valeur ne doit être que le préambule à de nouvelles évolutions et de nouveaux changements. Cette dynamique d'innovation doit être poursuivie pour maintenir le PM au sein du programme officiel des JO. La fédération internationale et les fédérations nationales de pentathlon moderne se questionnent actuellement pour proposer de nouvelles innovations ${ }^{1}$.

Cet article pose la question de la capacité qu'une organisation a de mettre en place une stratégie d'innovation de manière dynamique. L'UIPM est-elle en mesure successivement d'innover stratégiquement?

Pour répondre à la question de recherche le cadre théorique de l'innovation-valeur sera présenté. Une méthodologie qualitative par étude de cas est utilisée. Des entretiens d'experts (56), des données secondaires au sein des différentes organisations (CIO, CNO, UIPM, FFPM) et dans la presse, spécialisée et généraliste, ont été collectés. Les résultats obtenus seront présentés et discutés. Répondre à cette question de recherche permettra à des organisations comme l'UIPM de poursuivre une stratégie d'innovation-valeur. Cette recherche essaiera de savoir si l'innovation-valeur peut être une dynamique stratégique.

\section{L’Innovation Stratégique «Océan Bleu»}

Les « océans bleus» représentent des espaces vierges possibles grâce à la créativité des dirigeants capables de réaliser de l'innovation-valeur. Ils sont en opposition aux «océans rouges », univers concurrentiel dans lequel les firmes s'affrontent pour détenir un avantage concurrentiel. Les auteurs proposent quatre possibilités, pouvant être complémentaires, pour une telle innovation : l'addition, l'élimination, la diminution ou le renforcement d'un facteur de l'offre proposée aux clients. Ces possibilités doivent créer une augmentation de la valeur pour ceux-ci tout en y combinant une diminution des coûts.

Plusieurs auteurs (Kim et Mauborgne, 2005; Voelpel et al., 2005; Hamel, 2008) expliquent que la rupture n'est pas obligatoirement technologique et que la création d'une innovationvaleur peut se réaliser à partir du produit ou du service existant (Lehmann-Ortega et Roy, 2009). L'innovation-valeur ne remplace pas systématiquement le marché existant mais peut soit le remplacer, soit coexister avec celui-ci. Pour que l'innovation stratégique voit le jour, elle peut s'appuyer sur six leviers que sont : 1. la modification de la conception que se font les managers des substituts, 2. la modification des groupes stratégiques, 3. une modification de la chaîne des acheteurs, 4 . une proposition de produits et services complémentaires, 5 . une modification des habitudes des clients et 6 . une compréhension des tendances à venir au sein de l'industrie (Kim et Mauborgne, 2005).

Pour mettre en place la stratégie océan bleu, les auteurs vont proposer une grille de quatre actions :

Cet outil recherche simultanément la différenciation et la domination par les coûts. Il ne se concentre pas uniquement sur la création, au risque de tomber dans le piège de la sophistication à outrance (Miller, 1993).

\section{FIGURE 1 \\ Stratégie d'innovation-valeur «Océan Bleu»}

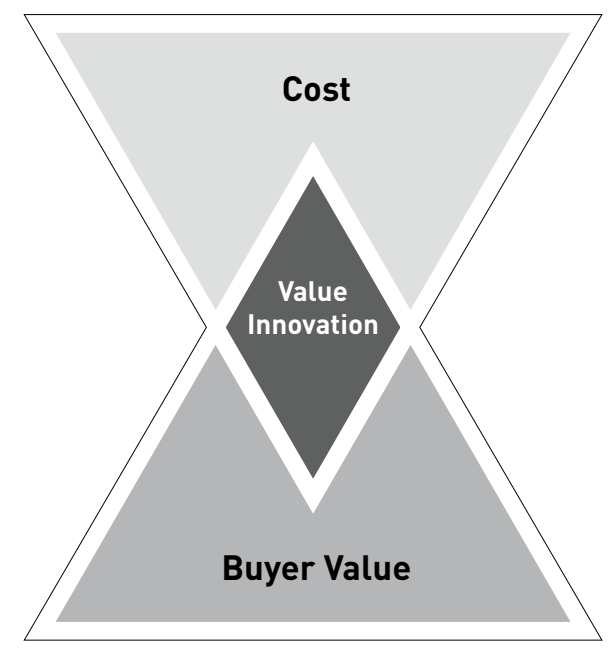

Source : Kim et Mauborgne, 2005

\begin{tabular}{|l|l|}
\multicolumn{2}{|c|}{ FIGURE 2} \\
\multicolumn{1}{|c|}{ La grille des 4 actions } \\
\hline Exclure & Renforcer \\
\hline $\begin{array}{l}\text { Quelles caractéristiques, qui } \\
\text { sont fondamentales pour le } \\
\text { secteur et les concurrents, } \\
\text { ne créent pas de valeur et } \\
\text { doivent être exclues? }\end{array}$ & $\begin{array}{l}\text { Quelles caractéristiques } \\
\text { doivent être renforcées } \\
\text { car sous estimées par } \\
\text { la pratique actuelle du } \\
\text { secteur? }\end{array}$ \\
\hline $\begin{array}{l}\text { Atténuer } \\
\text { Quelles caractéristiques } \\
\text { du produit ou du service ne } \\
\text { peuvent être supprimées, } \\
\text { mais dont l'importance } \\
\text { et la portée doivent être } \\
\text { atténuées? }\end{array}$ & $\begin{array}{l}\text { Quelles caractéristiques } \\
\text { absentes doivent être créées } \\
\text { afin de générer de la valeur } \\
\text { pour le client? }\end{array}$ \\
\hline
\end{tabular}

Source : Kim et Mauborgne, 2005 


\section{Méthodologie}

Cette recherche repose sur une méthodologie qualitative par l'étude de cas (Yin, 2003). Elle a l'avantage d'analyser un phénomène contemporain dans un contexte réel. Elle observe des phénomènes organisationnels cachés, tabous ou imperceptibles sans ces techniques (Reeves Sanday, 1979). Des concepts comme la culture, l'apprentissage ou l'innovation sont plus facilement évoqués durant des interviews. L'entretien permet la confiance et la restitution des notions recherchées (Blanchet et Gotman, 1992).

Des données primaires et secondaires ont été rassemblées pour les trianguler et s'assurer leur crédibilité (Miles et Huberman, 1985; Eisenhardt, 1989). Des entretiens d'experts (56) ont été réalisés en interrogeant des membres du CIO, des membres de CNO étrangers et français, des membres de la fédération internationale de PM et des membres de la fédération nationale, des membres de la direction technique nationale et des présidents de clubs français $(70 \%$ des clubs français ont participé à cette recherche). Ces entretiens ont été réalisés sur le territoire européen et durant l'année 2015. En plus des experts interrogés, les sportifs actuels ont été sollicités et $40 \%$ des pentathlètes français ont accepté de participer. Le but étant de leur demander s'ils avaient conscience des innovations passées et quel accueil ils feraient à de nouvelles innovations. L'entretien, élément central dans la collecte de données, était semi-directif. L'objectif, au travers de cinq thèmes de questions tournant autour des notions d'innovation-valeur (Exclure, Atténuer, Renforcer, Créer) devait permettre l'évocation de conditions spécifiques devant être soit repensées, soit changées, soit abandonnées, soit proposées.

Des documents internes (725 : rapports commissions CIO, rapports annuel CIO, Assemblée générale CIO..., rapports UIPM, règles disciplines UIPM..., rapports Ministère des sports, rapports FFPM...) ont été collectés. Une base de données, d'articles de la presse internationale et de la presse française, généraliste (The New-York Times...) et spécialiste (l’Équipe) a été créée (1473 articles de presse). Tous les documents et les articles ont été sélectionnés sur une période commençant en 2002, à l'annonce du CIO de se séparer de certains sports dans le programme olympique (baseball, softball, pentathlon moderne), et s'achevant en 2015, au moment de la rédaction de cet article. Toutes ces données permettent une saturation des informations indispensable pour vérifier la concordance des informations collectées.

Les données doivent être codées (Eisenhardt, 1989) avant d'être soumises à une analyse de contenu. Le codage, dans cette recherche, repose sur un dictionnaire créé, grâce aux écrits d'auteurs, et complété par les mots porteurs de sens identiques obtenant prèws de $96 \%$ de fiabilité (Miles et Huberman, 1985). Wordmapper et Alceste permettent l'analyse de données en utilisant des techniques complémentaires, l'une par classification descendante hiérarchique et l'autre par classification ascendante hiérarchique (Lassalle, 2011). Les résultats statistiques génèrent une représentation des masses et donnent ensuite accès aux détails.

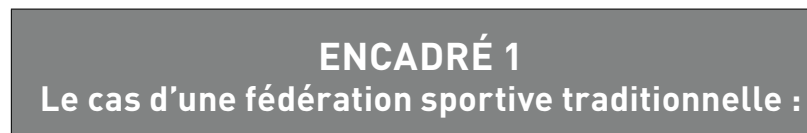

Pour la fédération française de pentathlon moderne, compte tenu de sa taille (plus petite fédération olympique), de son exposition médiatique (uniquement lors de J0), de son nombre de licenciés (environ 150), de ses ressources (921 000€ ${ }^{2}$ )... une approche directe du terrain se justifiait. L'accessibilité au terrain impose une démarche personnelle afin d'approcher les répondants, au travers de nombreux relais, et permettre l'évocation d'un sujet sensible comme l'innovation (Ramananstoa et Thiéry-Baslé, 1989). Cette recherche s'appuie sur des entretiens d'experts, sélectionnés dans les sphères les plus hautes des organisations en question (CIO, CNO, CNOSF, UIPM, FFPM... au travers de présidents, de directeurs généraux, de secrétaires généraux...). Pour s'assurer une forte implication des répondants, dans un environnement encore peu analysé comme les petites fédérations, la méthodologie se voulait au plus proche du terrain.

\section{Le pentathlon moderne}

Le pentathlon moderne et sa fédération internationale ont été retenus dans cette recherche pour tester la dynamique d'innovations-valeurs. Le Comité International Olympique exerce une pression plus forte, entre chaque olympiade, sur l'ensemble des sports présent ${ }^{3}$. La première explication réside dans les nouveaux enjeux financiers de l'organisation suisse. L'exposition médiatique de chaque discipline revêt un enjeu majeur. La logique commerciale prévaut face aux principaux diffuseurs. La deuxième explication est le nombre d'athlètes présents sur le site des JO et le village olympique. Depuis 20 ans, les JO ont connu une inflation du nombre de disciplines et de sportifs présents, entrainant une complexification de la gestion du village, des chambres, du planning des disciplines... (Lardinoit et Tribou, 2004). La troisième explication est l'enjeu lié, à une présence aux JO, pour chaque discipline dans son développement. Pour certains sports, comme l'aviron ou l'escrime, une présence aux JO reste indispensable afin de promouvoir les disciplines (épée, sabre et fleuret pour l'escrime). Cette exposition est essentielle pour attirer et fidéliser les pratiquants qui sont les clients de ces sports. Sans pratiquant une discipline disparait. Il est donc important pour les fédérations internationales de pouvoir promouvoir leurs sports et disciplines.

Ces menaces ont conduit les dirigeants nationaux (FFPM) et internationaux (UIPM) à une réflexion d'innovation stratégique (Kim et Mauborgne, 2005). Durant les dernières assemblées générales du CIO, l'UIPM a revendiqué sa place au sein du programme officiel en partie grâce à sa filiation avec le Baron Pierre de Coubertin. Cette référence au créateur des jeux olympiques modernes est importante pour comprendre la place de ce sport dans le plus grand événement sportif international. Son maintien réside également dans le fait qu'environ $90 \%$ de ses revenus proviennent du CIO et de sa participation aux JO d'été (Source : FFPM et CNOSF). Sans cette présence, cette fédération et ce sport disparaîtraient. Aujourd'hui, bien que le CIO ait assuré le développement du PM, il lui demande

2. Revenus 2015 se décomposant de la manière suivante : $62,8 \%$ de subventions du Ministère des Sports, $23,1 \%$ d'événements, $4 \%$ des licences, $1,7 \%$ de partenariats. Le reste étant le résultat de vente d'équipements ou de subventions diverses. (Source : Rapport du commissaire aux comptes sur les comptes annuels de la FFPM 2015).

3. France24, 9/12/2014, « Le CIO réforme les JO ». 
FIGURE 3

Méthodologie de la recherche

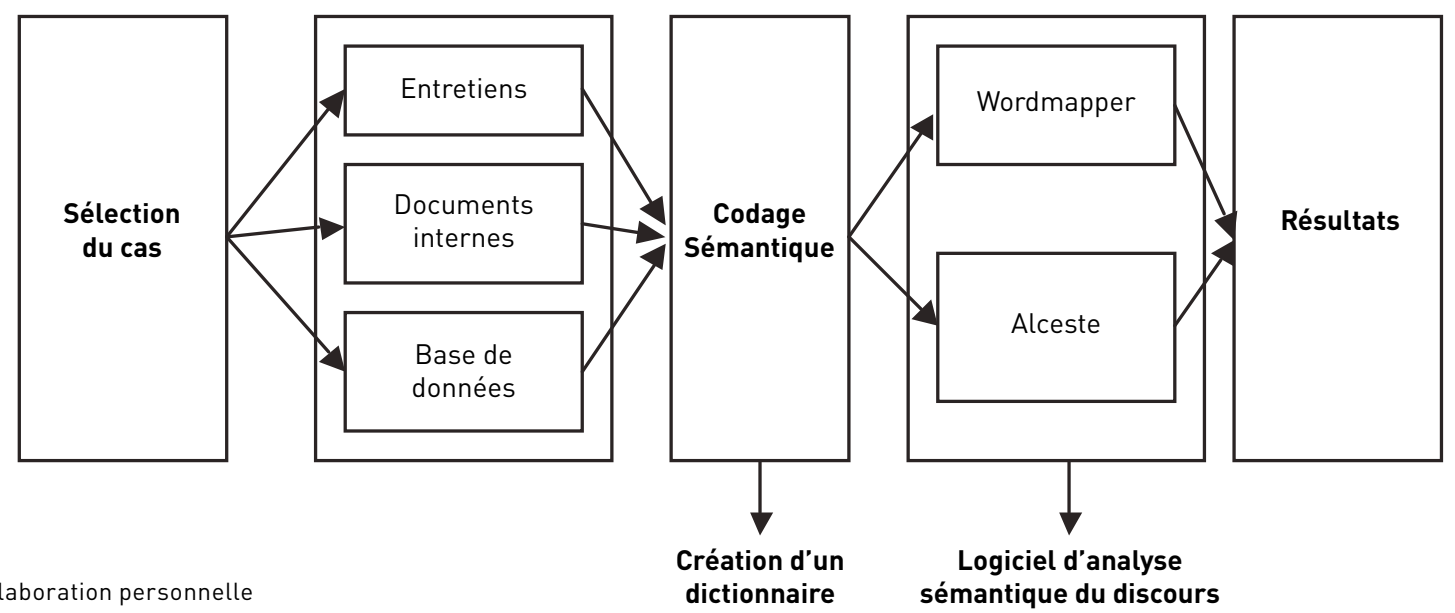

d'innover pour se maintenir dans le programme olympique en respectant ses trois contraintes (exposition médiatique, nombre d'athlètes, développement de la discipline). L'UIPM a déjà, par le passé, innové sur le matériel et sur le déroulement des épreuves lors des compétitions. Le pentathlon moderne est composé de quatre épreuves, mais de cinq sports, que sont l'escrime, l'équitation, la natation et un combiné (course à pied et tir au pistolet).

$\mathrm{Au}$ programme officiel des JO, seul le pentathlon moderne est présent. Le déroulement d'une compétition de PM est difficile à appréhender pour des non-initiés car le résultat intermédiaire entre chaque épreuve est complexe et les règles qui régissent ces résultats le sont également. Au 100 mètres nage libre, de natation ou d'athlétisme, le premier arrivé est déclaré vainqueur. Le vainqueur de l'escrime ou de l'équitation en PM n'est pas forcément bien classé au classement final (annexe 1). Par conséquent, un pentathlète doit calculer son investissement dans chaque épreuve en fonction de ses forces et faiblesses, et des barèmes, pour réaliser son enchainement d'épreuves. Cette évaluation permanente dans l'attribution des points peut rendre la compréhension difficile pour un nouveau pratiquant.

\begin{tabular}{|c|}
\hline $\begin{array}{l}\text { ENCADRÉ } 2 \\
\text { La fédération internationale comprend } \\
\text { aujourd'hui six disciplines : }\end{array}$ \\
\hline $\begin{array}{l}\text { 1. Le pentathlon moderne (PM); } \\
\text { 2. Le tetrathlon (4 épreuves avec l'équitation en moins } \\
\text { par rapport au PM); } \\
\text { 3. Le triathlé (3 épreuves avec l'escrime et l'équitation } \\
\text { en moins); } \\
\text { 4. Le biathlé (idem que le triathlé sauf que les épreuves } \\
\text { s'enchainent sans pause); } \\
\text { 5. Le laser-run (course et tir uniquement); } \\
\text { 6. Le biathlon à l'école (course et natation uniquement, } \\
\text { mais uniquement dans le cadre du programme scolaire } \\
\text { de certains pays). }\end{array}$ \\
\hline
\end{tabular}

Dans une logique d'innovation-valeur, l'UIPM cherche à faire encore évoluer son offre. Les résultats doivent permettre de savoir si l'UIPM peut encore innover stratégiquement. Quelle direction doit-elle prendre, compte tenu des attentes des deux principales parties prenantes, que sont le $\mathrm{CIO}$ et les pentathlètes (clients) eux-mêmes?

\section{Résultats}

Les résultats vont être présentés et illustrés avec des propos de personnes interviewées et de données collectées. La grille des 4 actions permet d'identifier, dans les données, les différents éléments qui peuvent favoriser une innovation stratégique. Concernant les caractéristiques de la grille, voici les propositions obtenues grâce à l'analyse des résultats.

\section{EXCLURE}

1. L'élément principal est l'échelle de notation des 4 épreuves. Bien que la combinaison de la dernière épreuve (Tir - Course) soit plus compréhensible du fait de l'ordre de départ et de la désignation du vainque ur final, les trois autres épreuves sont incompréhensibles pour des non-pentathlètes. Pratiquement tous les experts $(90 \%)$ et les pentathlètes $(86 \%)$ rencontrés ont évoqué cette difficulté.

[Monsieur D] : "L'échelle de notation, pour être honnête est difficile à comprendre, voir incompréhensible... Le ratio de victoire en escrime, les secondes en natation qui donnent des points, ou encore les difficultés de l'équitation ne permettent pas de suivre facilement une compétition. "

[Monsieur T]: "A chaque fois on se fait expliquer les barèmes car il faut une véritable calculette dans le cerveau pour suivre les différentes épreuves. L'avantage du combiné c'est que le premier est devant et que s'il ne se fait pas rattraper il gagne la compétition. C'est plus intéressant pour le suspense. Il faudrait arriver à trouver la même chose pour les autres épreuves. » 
[Monsieur W] : "On essaie de changer ce barème de notation mais c'est compliqué d'abandonner un système que tous les pentathlètes connaissent pour un autre système qui n'a pas encore fait ses preuves."

[Pentathlète 5] : «Au début, pour les premières compétitions, je ne comprenais pas les points. C'est mon entraineur qui me disait où je me situais. C'est en connaissant les autres compétiteurs et à force de faire des compétitions que l'on sait comment évoluer sur notre journée d'épreuves."

2. Il existe un problème lié à l'existence de $\mathbf{5}$ sites d'entrainement (30\% d'évocation). Pour les experts, un sport multi-sites contraint fortement les pentathlètes et rebutent les futurs pratiquants. Il est donc important de regrouper les disciplines afin de gagner en cohérence auprès des pratiquants.

[Madame AQ] : "Le multi-site est un problème non seulement pendant les semaines d'entrainement avec des parents qui doivent faire des kilomètres pour accompagner les enfants, mais également lors des compétitions car il faut en permanence se déplacer, reprendre la préparation, s'occuper des équipements, tout en essayant de maintenir nos pentathlètes dans la compétition.»

[Madame AE] : "Le pentathlon avec sa logique multi-site est devenu trop coûteux pour organiser des compétitions. A l'heure actuelle, en Europe seuls les pays de l'Est qui dominent la discipline organisent encore des compétitions. Afin d'attirer plus de sportifs, il faut relancer les compétitions de proximité et avec le multi-site c'est devenu impossible.»

[Monsieur AT] : "Il est compliqué de faire venir de nouveaux jeunes dans nos clubs parce que les parents bien que trouvant ce sport intéressant sont rebutés souvent par des lieux d'entrainement éloignés. En semaine nous avons réglé le problème en répartissant les entrainements de chaque discipline sur un jour à chaque fois, mais pour certains c'est encore trop complexe surtout au regard d'autres sports comme la natation qui se déroule à chaque fois au même endroit.»

3. Le dernier élément devant être exclu est un problème de gestion du temps pour l'organisation de la pratique. Pour $30 \%$ des pentathlètes, il s'agit d'un problème pouvant amener à l'abandon de ce sport. Pour les experts, cette gestion du temps entre les disciplines est aussi problématique en situation de compétition pour garantir une bonne performance des pentathlètes.

[Pentathlète 12] : "Le multi-site pose un problème de performance. Contrairement au triathlon qui enchaine les épreuves nous on doit s'arrêter entre chacune. C'est l'élément qui me gène le plus et je pense que les performances seraient meilleures s'il n'y avait pas tous ces arrêts. "

[Monsieur B] : "Il est impératif de rendre le pentathlon moderne plus cohérent dans son déroulement afin de faciliter sa diffusion et l'implication de tous les partenaires. Les expériences passées nous ont montré que les premières épreuves étaient délaissées par les spectateurs. Les médias ont aussi souhaité diffuser les derniers moments de la compétition au détriment des premières épreuves. Il est donc impératif de lier de manière plus clair et lisible ces 4 épreuves. La maîtrise de l'enchainement des épreuves et une meilleure gestion du temps doivent permettre de trouver des solutions.»
[Monsieur Z] : «Nous réfléchissons à un meilleur enchainement des épreuves. Il est difficile d'imposer aux pentathlètes et aux partenaires médiatiques et spectateurs trop d'attente entre les épreuves du fait d'un changement de site. L'escrime pourrait être une phase de sélection et les autres épreuves pourraient se regrouper sur un même site ce qui diminuerait le temps. »

\section{Atténuer}

1. Pour la majorité des personnes interrogées (experts, clubs, pentathlètes), la référence au Baron de Coubertin est trop présente dans ce sport (60\%). Cette évocation historique donne une image dépassée de ce sport et nie les nombreuses évolutions qui le rendent moderne. Cette filiation avec le créateur des Jeux Olympiques modernes bien qu'ayant une utilité au niveau des instances internationales, n'est plus en adéquation pour attirer des jeunes sportifs qui le plus souvent ne connaissent pas cette personnalité. Pour les experts, des références à la chevalerie ou à la piraterie sont porteuses de sens beaucoup plus fort pour les jeunes pratiquants.

[Monsieur AD] : "Au niveau du CIO, dans le passé, on a beaucoup joué sur notre relation avec le créateur des Jeux Olympiques modernes qui avait introduit le PM. Et par conséquent, les institutions et les clubs ont beaucoup communiqué là-dessus. Aujourd'hui, c'est un petit peu dépassé. Plus aucun jeune ne sait qui est le Baron Pierre de Coubertin.»

[Monsieur AP] : «Le Baron Pierre de Coubertin est inconnu de nos pentathlètes et pour être honnête on ne cherche plus à leur expliquer. C'est plus les parents qui posent les questions ou qui le disent à leurs enfants. "

[Madame AZ] : "Nous ça fait longtemps que l'on a arrêté de parler de de Coubertin. Les pentathlètes ce qui leur plait c'est le pistolet, l'épée, le cheval... et les parents c'est que ce soit un sport complet.»

[Monsieur AX] : "Nous n'avons jamais eu autant d'inscrits que depuis que nous utilisons l'image des pirates. Le film "Pirates des Caraïbes» nous a bien aidé. Lorsqu'on explique aux potentiels nouveaux adhérents que le pentathlète fait tout comme un pirate ils sont emballés. Aussi bien les filles que les garçons.»

[Pentathlète 3] : "Moi quand j'ai commencé je ne savais pas qui était de Coubertin ni son rapport avec le PM. Mes parents ont voulu que je fasse du PM parce que c'était un sport complet et qu'il n'y avait pas beaucoup de pratiquants. Moi ça me plaisait bien parce que je me prenais pour un chevalier avec mon cheval et mon épée."

[Pentathlète 10] : "J'ai découvert le PMà un salon des sports de ma ville. Le stand de démonstration était beau avec l'entraineur en pirate. J'ai pu tester le pistolet à plomb à l'époque et faire un peu d'escrime. Je me suis inscrits quelques jours après. Pour mes copains je dis que je fais le sport des pirates parce que le PMils ne savent pas ce que c'est...»

2. Le PM est un sport où la pratique est difficile $(63 \%$ des personnes interrogées). Cette difficulté réside dans la complémentarité de chacune des épreuves s'appuyant sur des 
capacités différentes (tir = concentration; course = endurance; natation = endurance; escrime $=$ technique; équitation $=$ technique et dressage). Un sport comme le triathlon (natation, cyclisme sur route et course à pied), pour les experts, est plus homogène car il est basé sur l'endurance.

[Monsieur A] : «Le PM est un sport très dur. Il faut mâेtriser 5 sports qui sont très différents. D’ailleurs chacun des sports est présent indépendamment aux JO. Pour le pentathlète, la maîtrise de ces disciplines pendra plusieurs années. D'autres sports comme le football ou le cyclisme semblent plus accessibles aux futurs sportifs. "

[Madame AA] : "Le complexité du PM dans la pratique est un frein pour attirer de nouveaux pratiquants. Beaucoup de ceux qui ont arrêté évoquent cette difficulté. La plupart pratiquent encore un des 5 sports après leur abandon $d u$ PM. Il est difficile avec des sports aussi hétérogènes de satisfaire tout le monde."

[Monsieur AG] : "Nous avons plusieurs formules avant d'arriver au PM. Le triathlé, par exemple, permet à nos adhérents ne commencer par 3 disciplines avant éventuellement de passer à 4 et 5. Cette offre de sport est importante pour satisfaire nos jeunes et éviter de les décourager avec le PM. »

[Monsieur AQ] : "Les entrainements pour les nouveaux adhérents et les jeunes sont forcements limités à deux ou trois disciplines. L'escrime et l'équitation arrivent dès que le pentathlète est suffisamment fort. Seuls les meilleurs pourront pratiquer le PM. Le Laser-Run est un bon début dans l'univers du PM.»

[Madame AL] : «Le PM est un sport difficile, mais c'est en même temps ce qui plaît au CIO. Un sportif complet capable de maîtriser 5 sports différents, c'est une vraie richesse pour le sport. Malgré tout, c'est vrai que cette difficulté rebute et est difficile à mettre en valeur médiatiquement. "

[Monsieur BD] : "Beaucoup de nos adhérents viennent principalement de la natation. Parmi ceux-ci, la plupart trouvent que le PM est trop dur et pourtant la natation n'est pas un sport simple. Avec les nouveaux adhérents on essaie au maximum de varier les entrainements, les exercices, la pratique pour casser la monotonie et faire accepter lentement la difficulté de pratique du PM.»

\section{RENFORCER}

1. Pour la plupart des acteurs de l'univers sportif, le pentathlon moderne possède des qualités. Pour les pentathlètes, il n'est pas assez perçu comme un sport évolutif (50\% des experts le pensent). En effet, l'UIPM est constituée de plusieurs «produits» très peu connus comme le biathlé ou le triathlé.

[Monsieur E] : «Le problème du PM c'est que personne ne sait ce que c'est. La natation tout le monde sait, l'escrime idem, l'équitation idem, le tir idem, la course à pied idem. Si on demande aux gens les possibilités qu'offre l'UIPM personne ne saura qu'il existe le biathlé ou le triathlé..."

[Monsieur H] : "Le PM c'est un beau sport. Attention! Dur et complet! Mais beau. Je pense que le Laser-Run peu permettre d'attirer des nouveaux sportifs. Le problème c'est que peu savent qu'ils peuvent progresser par étape avant de passer au PM. L'image qu'on les gens c'est qu'il faut tout de suite maîtriser ces 5 disciplines. "

[Monsieur L] : «Le PM et sa fédération font partis de la famille du sport français. Malheureusement, pour le grand public, seule l'épreuve "reine» existe grâce à sa présence aux JO. Il faut maintenant essayer de faire passer le message que la FFPM c'est une multitude de possibilités. La Fédération Française de ski et la fédération internationale $y$ sont arrivées avec toutes les disciplines (ski alpin, ski cross, ski acrobatique, saut...) pourquoi pas l'UIPM.»

[Monsieur X] : "Il faut que l'UIPM fasse des efforts pour proposer plus de compétitions de biathlé ou triathlé. Qu'on arrive à proposer aux clubs des formats plus attractifs. Pour le moment la formule n'est pas satisfaisante mais en même temps nous avons tellement de travail pour aider les clubs existants et aider à la création de nouveaux clubs. Il va falloir qu'on arrive à traiter l'ensemble de nos "produits» comme des produits à part entière et non pas comme une étape vers le PM.»

[Pentathlète 23] : "Je n'ai jamais pratiquéle PM, parce que je n'ai pas encore pu pratiquer les 5 disciplines en même temps. Mais je m'amuse bien avec mes trois épreuves et je n'ai pas forcément envie d'aller vers les 5. Le format de 3 me convient bien et me permet de m'investir totalement dans les 3.»

2. Il s'agit d'un sport complet (37,5\%). L'UIPM doit mettre plus en avant les qualités de ses disciplines et l'intérêt pour le client de le pratiquer.

[Monsieur M] : «Il faut rappeler qu'à Saint-Cyr il n'y a pas si longtemps tous les élèves pratiquaient le PM. C'est certes un sport historique, utile aux officiers de l'armée jadis, mais c'est encore et toujours un sport complet, parfait pour la formation du corps et de l'esprit. Malheureusement, le grand public ne le sait que peu faute d'exposition médiatique, de moyens, de clubs sur le territoire..."

[Madame S] : "Le PM c'est un super sport. Il faut être un sportif accompli, complet, endurant pour pouvoir le pratiquer. Son problème c'est que personne ne le connaît, ainsi que la complémentarité des disciplines. On a tendance à croire qu'il faut être jeune et potentiellement «olympisable» pour le pratiquer alors tout le monde peut le faire. En ce moment c'est le boum du trail, des triathlons et autre sport de nature. Les sportifs n'ont pas peur de faire des efforts quelque soit leur âge alors pourquoi pas du PM.»

3. $71 \%$ des pentathlètes sont heureux de pratiquer un sport où ils prennent du plaisir (30\%) du fait de la variété des épreuves et des entrainements. Il est donc nécessaire de renforcer ce niveau de variété pour maintenir une bonne satisfaction des pratiquants et des futurs pratiquants.

[Monsieur AS] : "Aux entraînements, les jeunes et les moins jeunes prennent du plaisir même si c'est parfois dur. Ceux qui arrêtent ce sport c'est soit parce que les copains font un autre sport, soit parce qu'ils n'ont plus temps, soit parce $q u$ 'ils veulent se recentrer sur un sport. Je n'ai jamais vu un pentathlète qui arrêtait parce que c'est un sport chiant. Avec l'expérience, on sait quand changer les exercices, les jeux, les directives pour finalement qu'ils soient toujours 
surpris bien qu'ils fassent toujours le même sport. C'est vrai que c'est plus facile à faire au PM que dans d'autres sports du fait de nos 5 disciplines. »

[Pentathlète 14] : "Moi je suis très heureux de faire du PM. J'ai fait du tennis et du foot avant mais ce n'était pas aussi varié. Parfois quand on fait natation et que j'en ai marre je me dis que le prochain entrainement ca sera tir ou course et du coup ca va mieux. Au foot c'est tout le temps foot...»

4. Le nombre de compétition doit être renforcé.

[Monsieur AC] : "Il faut qu'on arrive à développer de nouvelles compétitions, de nouveaux formats. C'est impératif pour créer une dynamique chez les pentathlètes. Le rêve serait un circuit professionnel comme au golf ou au tennis. Mais pour arriver à ça, il faut développer des compétitions en local, en national, en continental et en international. Les compétitions de PM coûtent trop chères aux organisateurs, ils faut donc qu'on arrive soit à diminuer ce coût soit à proposer des choses différentes pour proposer plus de choses. »

[Madame AF] : "Les compétitions c'est le sujet qui fâche... Au niveau national on peut organiser une ou deux épreuves par an mais en local c'est impossible compte tenu du nombre de clubs et de la distance entre eux et des moyens dont ils disposent. Le club d'Aix-en-Provence ne peut pas envoyer ses jeunes tous les dimanches à Bordeaux, Rennes, Paris... Et les jeunes de Rennes n'ont pas tellement envie de faire des compétitions qu'entre eux... Pour avoir plus de compétitions, il faut plus de clubs.»

5. L'UIPM doit renforcer sa communication afin d'obtenir une reconnaissance médiatique. Cette absence médiatique est un problème pour $77 \%$ des experts et pour $68 \%$ des pentathlètes.

[Monsieur AH] : "Notre principale faiblesse c'est que personne ne connaît le PM parce que personne ne parle du PM. On participe pourtant à tous les salons sportifs, aux démonstrations, on a un site internet mais le seul éclairage que l'on a vraiment c'est la quinzaine olympique et encore quand France Télévision nous diffuse. On ne sait pas vraiment comment communiquer pour qu'on s'intéresse à nous. On n'a pas su capitaliser sur Amélie Cazé par exemple, notre Teddy Riner à nous.»

[Madame AJ] : "On le sait aujourd'hui pour qu'un sport existe la télé est primordiale. On l'a bien vu avec le développement du rugby avec Canal+. Il faut une présence visuelle plus forte pour le PM mais peut être qu'elle ne doit pas passer par les chaînes traditionnelles. Internet aujourd'hui offre d'incroyables possibilités. Youtube et les chaînes que l'on peut y créer doivent nous amener à réfléchir à notre communication."

[Pentathlète 27] : "J'ai découvert le PM par hasard (salon des sports). Je ne savais pas que ça existait avant et je ne savais même pas que c'était aux JO ou à la télé. C'est compliqué de trouver des vidéos pour les montrer à mes copains pour qu'ils comprennent ce que je fais. Eux pour le foot ou le rugby, c'est plus simple...»

\section{Créer}

1. Le premier élément à créer est lié à l'échelle de notation. Une nouvelle échelle simple doit voir le jour (60\% des experts le pensent). Compte tenu d'épreuves d'affrontement (escrime) et de vitesse (natation, course - tir, équitation), il est cohérent qu'il coexiste deux échelles de notation.

[Monsieur I] : "Le PM c'est incompréhensible! Non pas les sports mais le classement, les points. L'escrime avec ce ratio de point c'est une horreur. Il faudrait que l'UIPM mette en place une sorte de tableau comme au Tennis. Le vainqueur du tournoi d'escrime à un bonus de temps par exemple. On retrouve le même problème avec la natation qui pourrait se régler facilement. L'équitation c'est un peu plus complexe mais ça doit être possible. De toute façon actuellement c'est incompréhensible alors ils ne peuvent que faire mieux. "

[Madame AI] : "On sait que l'échelle de notation est un problème, mais aujourd'hui en créer une nouvelle est aussi un problème pour sa complexité. En l'état actuel des épreuves et des disciplines, on voit mal comment changer cette échelle. Mais on est conscient qu'elle doit changer c'est juste que c'est aussi une discussion que doit avoir l'UIPM et ca ne se joue pas qu'au niveau de la FFPM.»

[Monsieur O] : «La FFPM doit être force de proposition dans le changement de cette échelle de notation. On a une chance d'avoir des clubs prêts à tester de nouvelles formules, il faut que la FFPM et le sport français devienne un laboratoire d'idées et d'innovations pour peser dans l'avenir du sport. Et pour être honnête qui comprend aujourd' hui le classement intermédiaire et les points au PM. Il faut faciliter la vie du pentathlète, du spectateur et du diffuseur avec une nouvelle échelle simple.»

2. La création d'une vraie communication sur le pratique atypique de ce sport (47\%) est évoquée. Pour le moment, ce sport trop confidentiel n'est que l'accumulation de 5 sports, et n'est pas mis en avant comme un sport unique avec des forces évidentes capables d'attirer des clients.

[Monsieur N] : «Il est difficile aujourd'hui avec 4 épreuves séparées géographiquement, temporellement et en terme de notation, d'avoir une unité "sportive». Il est donc normal que pour le moment le PM apparaisse comme un sport d'accumulation d'autres sports que comme un sport à part entière. L'unité peut s'obtenir soit par la complétude du sportif soit par une refonte géographique, temporelle et en terme de notation. La communication peut aider pour la première possibilité.»

[Monsieur AU] : «Le PM a quand même de sacré argument pour lui. Il est complet, divertissant, atypique, il fait penser aux pirates, aux chevaliers, aux aventuriers. Il n'y a quand même pas beaucoup de sports qui peuvent proposer tout ça. Maintenant il faut le faire savoir aux jeunes, aux parents, aux médias."

3. Pour l'ensemble des experts, le Laser-Run pourrait parfaitement remplacer le PM au programme officiel des JO du fait de sa facilité d'exposition, de diffusion et de compréhension des spectateurs. Les pratiquants sont eux beaucoup plus réticents à un tel changement. 
[Monsieur K] : "L'avenir de l'UIPM passe peut-être par le Laser-Run. Le biathlon en hiver a parfaitement su trouver sa place aux JO. Il peut en être de même avec le LR. Le PM n'a pas su trouver une place malgré une présence ancienne aux JO d'été, il est temps soit de donner sa chance au LR soit de repenser intégralement et rapidement les épreuves et tout ce qui fait le PM. »

[Monsieur G] : "Le PM c'est dépassé. C'est le sport des officiers de l'armée. Est-ce qu'on a envie encore de cette image aux JO? Le LR surtout avec sa technologie laser c'est l'avenir, la modernité et ce vers quoi doit tendre le CIO.»

[Pentathlète 6] : "Si le LR devenait olympique je ne serai pas très content. Pour nous les pentathlètes le graal c'est d'arriver à maitriser les 5 sports et si en plus on peut participer aux JO c'est le top. Par contre le LR ce n'est pas assez technique, pas assez dur et ça sera forcément moins sympa que de ne plus faire que de ça."

4. La création pour l'UIPM doit passer par une refonte des épreuves. Pour la majorité des experts et pour une grande partie des pentathlètes interrogés, l'esprit du pentathlon moderne est dans la pratique des 5 sports (escrime, natation, équitation, tir, course) mais pas dans les épreuves proposées.

[Monsieur Y] : "On doit s'interroger sur ce qu'est le PM. Parce que si nous ne le faisons pas, les autres (CIO, Médias, sportifs) le feront pour nous. Qu'est ce que nous voulons? Être dans la tradition de ce qu'a été le PM? Ou bien évoluer vers ce que nous pensons que le PM doit être? Beaucoup de sports ont évolué (rugby, natation). Un changement d'épreuves, de format, de notation peut parfaitement être envisagé pour transformer dans la bonne direction le PM.»

[Monsieur AB] : «Le PM a su évoluer ses dernières années pour répondre aux différentes demandes $d u$ CIO. Le combiné course-tir en est le meilleur exemple. Doit-on continuer? Oui. Doit-on changer complètement? Peut-être mais le changement prend du temps. Est-ce qu'une refonte totale des épreuves changerait la philosophie de ce sport? Je ne crois pas."

[Madame AO] : "Il faut que l'on continue nos réformes sur le PM. Le CIO doit voir nos efforts pour rendre plus attractif ce sport. En même temps en continuant dans cette direction je suis sûr que l'on va attirer de plus en plus de sportifs dans nos clubs. »

[Madame AQ] : "Une refonte complète des épreuves irait dans le sens du CIO et dans les attentes des sportifs. On le voit bien que l'eau libre, le cross nature, les activités en extérieures séduisent de plus en plus. Et finalement est ce qu'une nouvelle combinaison ne serait pas plus légitime aux yeux de tous les partenaires. "

[Pentathlète 35] : "Entre une entrée du LR aux JO ou le maintien du PM avec d'autres épreuves, je préfère le maintien du PM. Parmi les combinaisons possibles une nage en eau libre, avec des enchaînements rapides entre nage, équitation et course à pied-tir ça serait parfait. Pour moi passer de la natation en piscine à de la nage en eau libre finalement ne change pas grand chose.»
[Pentathlète 18] : "Le PM pour moi c'est les 5 sports ensembles qui au fur et à mesure des années on essaie de maîtriser. Faire de l'équitation qu'il s'agisse de saut d'obstacles ou de cross, ça revient au même puisqu'on fait toujours de l'équitation. Nager c'est pareil en piscine ou en extérieur, donc un changement des épreuves si c'est pour gagner en spectacle je suis d'accord."

Les résultats présentés permettent d'identifier des éléments sur lesquels peut se mettre en place une dynamique stratégique d'innovation valeur pour l'UIPM. La partie suivante va permettre de discuter les éléments présentés.

\section{Discussion}

Les experts, ainsi que les données secondaires, évoquent des changements antérieurs dans le PM. Peu d'entre eux comprennent qu'il s'agit d'innovation. La catégorisation en processus, produit, stratégique ou managériale (Hamel, 2008), n'est pas appréhendée par les principaux dirigeants du sport mondial. Ils souhaitent changer certains éléments du PM en espérant trouver la bonne innovation mais sans analyse préalable. L'UIPM n'a pas d'approche structurée d'un processus d'innovation stratégique. Pour les pratiquants, seulement $67 \%$ d'entre eux ont connaissance des évolutions antérieures. Ils reconnaissent, une fois celles-ci expliquées, qu'elles ont été bénéfiques (89\%). Cette méconnaissance de leur propre sport s'explique, en partie, par le jeune âge des licenciés (14 ans d'âge médian des répondants chez les pentathlètes). Une autre raison est le peu d'antériorité dans la discipline (en moyenne, les pentathlètes répondants n'avaient que 4 ans de pratique de ce sport).

Le PM est confronté, compte tenu de ses deux principales parties prenantes, le Comité International Olympique et ses clients (pentathlètes licenciés), à des enjeux afin d'évoluer. Afin de garantir une innovation-valeur, l'UIPM doit garder à l'esprit qu'elle doit diminuer ses coûts tout en augmentant la valeur pour le client. Bien que le CIO soit une partie prenante importante, le client reste avant tout le sportif qui par sa pratique donne une légitimité au sport et une légitimité à sa présence au programme officiel des JO.

Dans cette perspective d'innovation-valeur, $80 \%$ des clients pensent qu'un changement d'épreuve est nécessaire afin de favoriser la création de valeur. Les résultats mettent en avant le problème posé par l'échelle de notation (à exclure et à créer). Compte tenu de la spécificité des épreuves (une d'affrontement et trois de vitesse), il est difficile d'avoir une vraie fluidité. Il est possible de la gagner sur les trois épreuves de vitesse. Pour $23 \%$ des clients interrogés, l'eau libre aurait toute sa place au lieu de la piscine. Ce changement permettrait une meilleure unité spatiale pour les épreuves et un enchainement plus rapide sur les épreuves de vitesse. Pour $75 \%$ des clients la combinaison idéale serait :

1. Le matin du jour de compétition : escrime. Le pentathlète commence l'épreuve avec un bonus/malus de temps de 0 minute. Chaque victoire donne des secondes d'avance et chaque défaite des secondes de retard.

2. L'après-midi : les pentathlètes s'élancent dans l'eau libre en fonction des secondes obtenues ou perdues à l'escrime. En sortant de l'eau, sans pause et chronométré, ils doivent 
réaliser un cross à cheval. En descendant du cheval, sans pause et chronométré, ils finissent par le cross-tir comme actuellement.

Ce format d'épreuve permet d'avoir une fluidité entre les épreuves de l'après-midi. Mis à part après la première épreuve (escrime), le leader serait facilement indentifiable. Il serait le premier à passer à l'épreuve suivante. Le vainqueur devra moins s'économiser sur les différentes épreuves et avec la disparation des échelles de notation actuelles moins calculer. Il gagnerait en visibilité par sa position dans les trois dernières épreuves (50\% d'adhésion dans les réponses). Cette innovation au sein des épreuves est acceptée car la disparition du PM au sein des JO détruirait la valeur pour les clients. Ils préfèrent cette refonte des épreuves à l'introduction du Laser-Run (25\% seulement de soutien pour ce dernier). L'acceptation du Laser-Run (42\%) n'existerait que si le CIO refusait tout autre possibilité. Malgré tout, pour $52 \%$ des clients cela signifierait un arrêt de la pratique du PM pour se concentrer sur un seul des cinq sports (principalement la natation).

Pour justifier que ce changement correspond à une innovation-valeur, il faut s'assurer que la grille des 4 actions est bien utilisée pour cette amélioration. Il faut vérifier également qu'il y a une diminution de coût et une augmentation de la valeur pour le client (Kim et Mauborgne, 2005). Concernant la diminution de coût, pour l'organisation et la pratique du PM, certaines épreuves n'ont pas été impactées. C’est le cas de l'escrime, de la course et du tir, qui se dérouleraient comme actuellement. Par contre, en ce qui concerne la natation et l'équitation, il y a des changements. Le passage pour la natation (absence de construction d'une piscine fermée et entretien) à une eau libre naturelle est moins consommateur de ressources. Pour l'équitation, les deux activités (saut d'obstacles et cross) ayant lieu en plein air, elles sont équivalentes en terme de consommation de ressources et de coûts. Pour le saut, les gradins temporaires sont disposés autour du parcours, là où pour le cross, ils seraient positionnés à des endroits clés du parcours. Cette évolution est dans la logique d'une innovation-valeur avec une réduction des coûts, essentiellement liée à l'épreuve de natation. D'autres coûts seraient également minorés (logistique, accueil, billetterie...).

Pour l'augmentation de valeur pour le client, la grille des 4 actions aide à démontrer les gains d'une telle refonte d'épreuves. Sur l'échelle de notation, le changement d'épreuves facilite l'implication, dans le déroulement de la compétition, du pentathlète. Elle gagne en clarté pour d'autres parties prenantes (spectateurs, média, téléspectateurs, CIO). L'échelle de notation de l'escrime gagne également en simplicité. Pour le côté «multi-sites», le problème ne se trouve réglé que lors de compétition ou finalement deux sites coexistent (l'escrime et les trois autres épreuves). Pour le client, le critère de multi-sites n'est pas résolu pour l'entrainement. Il en est de même pour la gestion du temps à l'entrainement. Cette continuité dans les épreuves peut ainsi renforcer l'image de sport complet que doit mettre en avant l'UIPM. En diminuant les coûts liés à la compétition, il devient plus facile d'organiser des compétitions. Cette augmentation des compétitions, avec le renforcement de la présence de sites naturels, contribue à l'augmentation de valeur pour le client. L'enchainement des épreuves offre à l'UIPM la possibilité d'homogénéiser le discours sur le fait qu'il s'agit d'une épreuve de vitesse (ou d'endurance), atténuant ainsi la disparité entre les cinq disciplines. Tous les éléments évoqués précédemment contribuent à de la création de valeur pour le client.

Compte tenu de la diminution de coûts et de création de valeur pour le client, une innovation-valeur est possible pour les organisations en charge du pentathlon moderne.

La démarche lancée, par l'UIPM depuis de nombreuses années pour innover stratégiquement, rend possible de nouvelles innovations. La recherche d'une innovation-valeur dans cet article se concentre sur le client légitime, le pentathlète. Les évolutions demandées par l'instance olympique ne peuvent être supportées par les instances fédérales que si elles sont en adéquation avec la consommation de ce sport par des pratiquants actuels, ou futurs. Une organisation sportive doit d'abord s'interroger sur sa capacité à survivre en dehors du programme officiel des JO avant même de savoir si elle est capable de s'y maintenir. Pour survivre, elle doit soit satisfaire son client actuel (les pentathlètes), soit chercher à gagner de nouveaux clients (les futurs pentathlètes).

Une des perspectives de recherche sera de tester à l'échelle d'un ou plusieurs clubs, d'une région ou même d'une fédération nationale, cette innovation-valeur afin de mesurer l'adhésion des clients et des non-clients. Un succès de ce nouveau format du pentathlon moderne pourrait ouvrir de nouvelles voies à l'UIPM. Une autre perspective serait de rechercher si une innovation managériale pourrait garantir une survie plus soutenable à l'UIPM. D'après la classification de Hamel (2008), l'innovation managériale est supérieure à l'innovation stratégique. Il serait donc intéressant de réfléchir à la possibilité, dans le cas du pentathlon moderne, d'une telle innovation.

Au niveau managérial, l'intérêt de cette recherche repose dans les préconisations que peut mettre en place l'UIPM. Cette recherche au travers de la stratégie «océan bleu» a démontré qu'une innovation-valeur était possible. Les instances fédérales peuvent donc essayer, au travers de la grille des 4 actions, de chercher des éléments permettant une innovation-valeur différente s'ils ne souhaitent pas changer le format des épreuves du PM. Dans une situation de menaces grandissantes, à la fois de la part du CIO et de la part des autres disciplines sportives qui souhaitent prendre une place au programme officiel des JO, l'UIMP et les fédérations nationales de PM doivent commencer à utiliser des grilles d'analyse stratégique d'innovation. Ces grilles peuvent être celle de Buaron (1981), Hamel (2008) ou de Kim et Mauborgne (2005), mais le plus important étant que les changements nécessaires à venir au sein du PM soient pilotés stratégiquement. Cette recherche a permis de démontrer l'utilisation et l'utilité d'une telle démarche pour les dirigeants des fédérations sportives.

Un autre apport réside dans l'absence d'étude sur l'innovation stratégique dans les organisations sportives. Parmi les écrits de la littérature sportive, la plupart des auteurs se sont concentrés sur l'étude de l'organisation d'un événement sportif au travers des fonctions organisationnelles (billetterie, logistique...). Les autres recherches ont mesuré l'impact de ces événements ponctuels, rares et recherchés, intuitivement générateurs de fortes retombées financières, médiatiques ou touristiques. Par contre, peu de recherches ont été menées sur l'innovation stratégique pour les organisations sportives. 


\section{Conclusion}

Cette recherche s'interroge sur la dynamique d'innovation stratégique d'une organisation. L'exemple du pentathlon moderne et de sa fédération internationale montre qu'il est possible d'avoir une dynamique d'innovation-valeur. Ainsi, la réponse à notre question de recherche est positive sur la capacité que peut avoir une organisation à innover plusieurs fois successivement. Les auteurs Kim et Mauborgne (2005) évoquent dans leur ouvrage, ou leurs articles, sur l'océan bleu, la capacité qu'une organisation a d'en créer. Ils ne démontrent pas, pour une même organisation, sa capacité à reproduire une innovation-valeur. Cette recherche permet d'y contribuer en affirmant que l'innovation-valeur comme dynamique stratégique est possible. Les recherches futures devront se demander s'il existe, dans cette dynamique, des freins internes ou des caractéristiques externes pouvant mettre un terme à celle-ci. Kim et Mauborgne (2005) avancent même qu'aucune organisation ne peut être dans un processus d'innovation-valeur permanent. "Comme les secteurs d'activités, les entreprises ont toutes leurs phases de grandeur et de décadence. En un mot, l'entreprise toujours gagnante n'existe pas plus que le secteur d'activité qui aurait éternellement le vent en poupe.» (Kim et Mauborgne, 2005, p. 220). Si une organisation peut se maintenir grâce à des innovations-valeurs successives alors l'approche océan bleu offre de nouvelles opportunités stratégiques. Pour cela, le déploiement de l'innovation stratégique est essentiel. Les managers des organisations doivent en permanence se demander comment une innovation-valeur peut redessiner les frontières de leur organisation. Ils ne doivent pas maintenir des règles immuables sous prétexte de tradition. La grille des 4 actions permet d'avoir une vision claire sur ce qu'est l'organisation et quels sont les clients.

Cet article a fait le choix de s'intéresser à l'innovation-valeur car c'est une innovation stratégique peu étudiée dans le cas des organisations sportives. Cet article s'est appuyé sur des experts des organisations et des pratiquants licenciés. Parmi les différentes parties prenantes (les sponsors, les médias (particulièrement les groupes télévisuels), les fédérations nationales, les spectateurs et pentathlètes), cette recherche a considéré que le pentathlète était, avec le CIO, la partie prenante la plus importante actuellement pour l'UIPM. L'approche d'Ansoff (1968) permet de comprendre que l'organisation doit réaliser des objectifs non seulement pour sa survie mais également pour satisfaire un jeu relationnel avec ses partenaires (clients (sportifs actuels), fournisseurs (médias), investisseurs (sponsors)). Mais quelle que soit la force de cette partie prenante, l'innovation-valeur doit se concentrer sur le client.

Pour le pentathlon moderne et la fédération internationale, l'avenir de ce sport, passera par une innovation. La question qui demeure est de savoir si cette innovation sera suffisante pour satisfaire le $\mathrm{CIO}$ tout en attirant plus de pratiquants.

\section{Bibliographie}

Ansoff, Igor (1968). Stratégie du développement de l'entreprise, Paris, Editions Hommes \& Techniques, 287 p.

BeCKer, Markus C. (2004). «Organizational routines : A review of the literature», Industrial and Corporate Change, Vol. 13, $\mathrm{N}^{\circ} 4$, p. 643-677.
Becker, Markus C.; Knudsen, Thorbjørn (2005). «The role of routines in reducing pervasive uncertainty", Journal of Business Research, Vol. 58, $\mathrm{N}^{\circ}$ 6, p. 746-757.

Birkinshaw, Julian; Hamel, Gary; Mol, Michael J. (2008). "Management innovation", Academy of Management Review, Vol. 33, $\mathrm{N}^{\circ} 4$, p. 825-845.

BIRKINSHAW, Julian (2010). Reinventing management, Chichester, John Wiley \& Sons Ltd, 289 p.

Blanchet, Alain; Gotman, Anne (1992). L'enquête et ses méthodes: l'entretien, Paris, Nathan, 128p.

Buaron, Roberto (1981). "New-game strategies», The McKinsey Quarterly, Vol. 12, N Spring, p. 24-40.

Burke, Andrew E.; Van Stel, Andre J.; Thurik, Roy (2009). «Blue ocean versus competitive strategy : theory and evidence», ERIM Report Series, Research in management, N $^{\circ}$ ERS-2009-030-ORG, p. 25.

Čirjevskis, Andrejs; Homenko, Genadijs; Lacinova, Valērija (2010). «New Approaches in Measuring and Assessing Viability of Blue Ocean Strategy in B2B Sector ", Journal of Business Management, $\mathrm{N}^{\circ} 3$, p. 162-179.

Cohen, Wesley M.; Levinthal, Daniel A. (1990). «Absorptive capacity : a new perspective on learning and innovation", Administrative Science Quarterly, N³5, p. 128-152.

Christensen, Clayton M. (1997). The Innovator's Dilemma, Boston, Harvard Business School Press, 288 p.

D’Amato, Alessia; Roome, Nigel (2009). « Toward an integrated model of leadership for corporate responsibility and sustainable development : a process model of corporate responsibility beyond management innovation", Corporate Governance : The International Journal of Business in Society, Vol. $9, \mathrm{~N}^{\circ} 4$, p. 421-434.

Damanpour, Fariborz; Aravind, Deepa (2012). «Managerial innovation : Conceptions, processes, and antecedents", Management and Organization Review, Vol. 8, $\mathrm{N}^{\circ} 2$, p. 423-454.

EisenhardT, Kathleen M. (1989). «Building theories from case study research", Academy of Management Review, Vol. 14, $\mathrm{N}^{\circ} 4$, p. $532-550$.

Feldman, Martha S. (2003). «A performative perspective on stability and change in organizational routines ", Industrial and Corporate Change, Vol. 12, $\mathrm{N}^{\circ}$ 4, p. 727-752.

GeBAuer, Heiko (2011). «Exploring the contribution of management innovation to the evolution of dynamic capabilities", Industrial Marketing Management, Vol. 40, № 8, p. 1238-1250.

Geroski, Paul (1998). "Thinking creatively about your market : crisps, perfume and business strategy», Business Strategy Review, Vol. 9, $\mathrm{N}^{\circ}$ 2, p. 1-10.

Grant, Robert M. (2008). «The future of management : Where is Gary Hamel leading us?», Long Range Planning, Vol. 41, № 5, p. 469-482.

Grodal, Stine; Nelson, Andrew J.; SiIno, Rosanne M. (2015). «Help-seeking and help-giving as an organizational routine : Continual engagement in innovative work", Academy of Management Journal, Vol. 58, $\mathrm{N}^{\circ}$ 1, p. 136-168.

Hamel, Gary; Prahalad Coimbatore K. (1989). «Strategic Intent», Harvard Business Review, Vol. 67, N³, p. 63-78. 
Hamel, Gary; Prahalad Coimbatore K. (1996). Competing for the Futur, Boston, Harvard Business School Press, 357 p.

HAMEL, Gary (2008). La fin du management : inventer les règles de demain. Paris, Vuibert, 249 p.

HARDER, Mie (2011). Internal Antecedents of Management Innovation, Copenhague, Copenhague Business School Press, 222 p.

Hoffman, Donna L.; Kopalle Praveen K.; Novak Thomas P. (2010). «The 'right' consumers for better concepts : identifying consumers high in emergent nature to develop new product concepts », Journal of Marketing Research, Vol. 47, $\mathrm{N}^{\circ}$, p. 854-865.

Kim, Chan W.; Mauborgne, Renée (2004). «Value innovation : The strategic logic of high growth», Harvard Business Review, Vol. 82, N $\mathrm{N}^{\circ}$ 7-8, p. 172-180.

Kim, Chan W.; Mauborgne, Renée (2005). Stratégie océan bleu : comment créer de nouveaux espaces stratégiques, Paris, Village mondial, $272 \mathrm{p}$.

Krinsky, Robert J.; Jenkins, Anthony C. (1997). «Collide : When the uneasy fusion of strategy and innovation», Strategy \& Leadership, Vol. 25, $\mathrm{N}^{\circ}$ 4, p. 37-41.

LARDINOIT, Thierry; Tribou, Gary (2004). «Quelle est la spécificité de la gestion du sport?», Revue Française de Gestion, Vol. 3, $\mathrm{N}^{\circ} 150$, p. $125-130$.

Lassalle, Frédéric (2011). «Proposition d'un outil d'analyse des relations de pouvoir sur les événementiels sportifs internationaux», Management et Avenir, $\mathrm{N}^{\circ} 39$, p. 52-64.

Lehmann-Ortega, Laurence; Roy, Pierre (2009). «Les stratégies de rupture», Revue Française de Gestion, Vol. 7, N 197, p. 113-126.

MArkides, Constantinos (1997). «Strategic innovation», Sloan Management Review, Vol. 38, №3, p. 9-24.

MARKIDES, Constantinos (1998). «Strategic innovation in established companies", Sloan Management Review, Vol. 39, N³, p. 31-42.

Martinsons, Maris G. (1993). «Strategic innovation : A lifeboat for planning in turbulent waters", Management Decision, Vol. 31, $\mathrm{N}^{\circ} 8$, p. 4-11.

Miles, Matthew B.; Huberman, Michael A. (1985). Qualitative data analysis, Newbury Park, CA : Sage, 337 p.

Miller, Danny (1993). Le Paradoxe d'Icare, Paris, Editions ESKA, $470 \mathrm{p}$.
Mol, Michael J.; Birkinshaw, Julian (2009). «The sources of management innovation: When firms introduce new management practices", Journal of Business Research, Vol. 62, $\mathrm{N}^{\circ} 12$, p. 1269-1280.

Mol, Michael J.; BirkinshaW, Julian (2012). «Relating management innovation to product and process innovation : Private rents versus public gains », in Pitsis T.S., Simpson A. and Dehlin E. (eds.), Handbook of organizational and managerial innovation, Cheltenham, Edwar Elgar, p. 13-35.

Nickell, Stephen; Nicolitsas, Daphne; Patterson, Malcolm (2001). «Does doing badly encourage management innovation?», Oxford Bulletin of Economics and Statistics, Vol. 63, $\mathrm{N}^{\circ}$ 1, p. 5-28.

O'Gorman, Patricio (2009). «Wii : Creating a Blue Ocean The Nintendo Way», Palermo Business Review, No 2, p.97-107.

Parvinen, Petri; Aspara, Jaakko; Hietanen, Joel; Kajalo, Sami (2011). «Awareness, action and context-specificity of blue ocean practices in sales management», Management Decision, Vol. 49, $\mathrm{N}^{\circ}$ 8, p. 1218-1234.

Ramananstoa, Bernard; Thiéry-Baslé, Catherine (1989). Organisations et fédérations sportives, Paris, Puf, 260 p.

Reeves-Sanday, Peggy (1979). «The Ethnographic Paradigm», Adminsitrative Science Quaterly, Vol. 24, p. 527-538.

Roy, Pierre (2007). «De l'intérêt des firmes dominantes à perturber et stabiliser le jeu concurrentiel», Finance Contrôle Stratégie, Vol. 10, $\mathrm{N}^{\circ}$ 3, p. 139-160.

Roy, Pierre (2010). Les Nouvelles stratégies concurrentielles, Paris, La découverte, $200 \mathrm{p}$.

Schlegelmilch, Bodo B.; Diamantopoulos, Adamantios; KreuZ, Peter (2003). «Strategic innovation : the construct, its drivers and its strategic outcomes », Journal of Strategic Marketing, Vol. 11, $\mathrm{N}^{\circ}$ 2, p. 117-132.

Voelpel, Sven; Leibold, Marius; Tekie, Eden; Von Krogh, Georg (2005). «Escaping the red queen effect in competitive strategy: Sense-testing business models», European Management Journal, Vol. 23, $\mathrm{N}^{\circ} 1$, p. 37-49.

Walker, Richard M.; Damanpour, Fariborz; Devece, Carlos A. (2010). «Management innovation and organizational performance : The mediating effect of performance management», Journal of Public Administration Research and Theory, $\mathrm{N}^{\circ} 21$, p. 367-386.

Yin, Robert K. (2003). Case study research : Design and methods, Thousand Oaks, Sage publications, $312 \mathrm{p}$. 


\section{ANNEXE 1 \\ Barème points épreuves de PM}

Le barème de notation des épreuves :

1. Escrime (Épée) : Tous les participants se rencontrent sur un assaut (combat) d'une minute ou en première touche gagnante. Si aucune touche n'est réalisée en une minute alors les deux participants de l'assaut ont perdu. Tous les concurrents se rencontrent dans une «poule unique». Pour obtenir 1000 points, il faut remporter $70 \%$ de victoires sur la totalité des assauts possibles.

2. Natation (200 mètres nage libre) : pour 2 minutes et 30 secondes, le pentathlète obtient 1000 points. Au delà ou en deçà du temps de référence chaque seconde vaut 12 points en plus ou en moins.

3. Équitation (Saut d'obstacles) : parcours de 12 obstacles et tirage au sort des chevaux qui appartiennent à l'organisateur de la compétition. Si le cheval et le cavalier font un sans faute laucune barre des 12 obstacles touchée) et respectent le temps imparti pour le parcours alors le pentathlète (cavalier) obtient 1200 points. Un barème est fixé pour la perte de points en fonction de chaque barre touchée, chaque refus et pour chaque seconde de retard sur la ligne d'arrivée du parcours équestre.

4. Combiné (Cross-tir) : il s'agit de la modification la plus récente du règlement du PM. L'épreuve de combiné consiste en une course de 3200 mètres ponctuée de 4 arrêts à un stand de tir où les concurrents utilisent un pistolet à tir laser et visent une cible située à 10 mètres. À chaque fois, le pentathlète doit toucher 5 cibles en un temps maximum de 50 secondes. Les concurrents sont classés selon leurs scores additionnés dans les trois premières disciplines (escrime, natation et équitation) et partent avec un handicap de temps, le leader partant le premier. Un point d'écart en moins, correspondant à une seconde de départ décalé pour le pentathlète suivant. De cette manière, la première personne à franchir la ligne d'arrivée est la gagnante de la compétition. 